\title{
Development and beam test of a continuous wave radio frequency quadrupole accelerator
}

\author{
P. N. Ostroumov, B. Mustapha, A. Barcikowski, C. Dickerson, A. A. Kolomiets, S. A. Kondrashev, Y. Luo, D. Paskvan, \\ A. Perry, D. Schrage, S. I. Sharamentov, R. Sommer, W. Toter, and G. Zinkann \\ Argonne National Laboratory, Argonne, Illinois 60439, USA \\ (Received 7 September 2012; published 14 November 2012)
}

\begin{abstract}
The front end of any modern ion accelerator includes a radio frequency quadrupole (RFQ). While many pulsed ion linacs successfully operate RFQs, several ion accelerators worldwide have significant difficulties operating continuous wave (CW) RFQs to design specifications. In this paper we describe the development and results of the beam commissioning of a CW RFQ designed and built for the National User Facility: Argonne Tandem Linac Accelerator System (ATLAS). Several innovative ideas were implemented in this CW RFQ. By selecting a multisegment split-coaxial structure, we reached moderate transverse dimensions for a $60.625-\mathrm{MHz}$ resonator and provided a highly stabilized electromagnetic field distribution. The accelerating section of the RFQ occupies approximately $50 \%$ of the total length and is based on a trapezoidal vane tip modulation that increased the resonator shunt impedance by $60 \%$ in this section as compared to conventional sinusoidal modulation. To form an axially symmetric beam exiting the RFQ, a very short output radial matcher with a length of $0.75 \beta \lambda$ was developed. The RFQ is designed as a $100 \%$ oxygen-free electronic (OFE) copper structure and fabricated with a two-step furnace brazing process. The radio frequency (rf) measurements show excellent $\mathrm{rf}$ properties for the resonator, with a measured intrinsic $Q$ equal to $94 \%$ of the simulated value for OFE copper. $\mathrm{An}^{5+}$ ion beam extracted from an electron cyclotron resonance ion source was used for the RFQ commissioning. In off-line beam testing, we found excellent coincidence of the measured beam parameters with the results of beam dynamics simulations performed using the beam dynamics code TRACK, which was developed at Argonne. These results demonstrate the great success of the RFQ design and fabrication technology developed here, which can be applied to future CW RFQs.
\end{abstract}

DOI: 10.1103/PhysRevSTAB.15.110101

PACS numbers: 29.20.Ej

\section{INTRODUCTION}

The new continuous wave $(\mathrm{CW})$ radio frequency quadrupole (RFQ) was developed to achieve higher efficiency and intensity for the ATLAS upgrade [1,2]. The RFQ will deliver $296.5 \mathrm{keV} / \mathrm{u}$ ion beams to the ATLAS positive ion injector linac (PII) with a very small longitudinal emittance formed by a four-harmonic buncher installed upstream of the RFQ. The first cryomodule of the PII will be modified to match the RFQ beams to the superconducting (SC) section; in particular, three small-aperture SC cavities will be removed from the PII cryomodule. These modifications will increase both the transverse and the longitudinal acceptance of ATLAS, while limiting the emittance growth for high-intensity beams. In addition, the californium rare isotope breeder upgrade (CARIBU) reaccelerated beam intensities will be increased by $40 \%$ at routine operations. The RFQ and associated bunching systems will provide $83 \%$ acceleration efficiency throughout ATLAS. In our application, the RFQ must provide stable operation over a wide range of rf power levels to allow the accelera-

Published by the American Physical Society under the terms of the Creative Commons Attribution 3.0 License. Further distribution of this work must maintain attribution to the author(s) and the published article's title, journal citation, and DOI. tion of ion species from protons up to uranium. The new ATLAS RFQ design incorporates developments that came out of the successful rare isotope accelerator (RIA) RFQ prototype tested in 2006 [3]. The resonator is a multisegment split-coaxial structure that reduces the maximum transverse dimension to 18 inches at an operating frequency of $60.625 \mathrm{MHz}$. The resonator structure is a $100 \%$ oxygen-free electronic (OFE) copper structure and is fabricated in a two-step furnace brazing process. This fabrication technology implies precise machining of the components before brazing such that no alignment is required afterwards. Previously, this technology has been successfully applied for the construction of a $350 \mathrm{MHz}$ RFQ known as the low energy demonstration accelerator (LEDA) at Los Alamos National Laboratory [4]. Until now, there is no experience in using brazing technology for construction of low frequency CW RFQs $(<200 \mathrm{MHz})$. For example, TRIUMF has developed, built, and successfully operates a $35 \mathrm{MHz}$ CW RFQ based on a split-ring 4-rod structure [5]. Because of the large dimensions of RFQ components, the rf power is managed very well, although the shunt impedance is greatly reduced due to multiple mechanical connections. There are other examples of unbrazed, low-voltage CW RFQs operated or being operated at Indiana University [6] and RIKEN Nishina Center [7] and Soreq Nuclear Research Center [8]. 


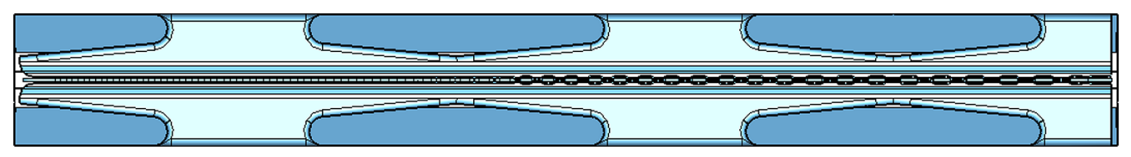

FIG. 1. Full 3D model of the RFQ in MW-STUDIO.

The main purpose of the paper is to report on the overall design, fabrication, and beam commissioning of the ATLAS RFQ. The detailed physics design of the RFQ and numerical and experimental beam dynamics studies including space charge effects will be presented in future publications.

\section{DESIGN}

\section{A. Physics and beam dynamics design}

The physics design concept of a heavy-ion RFQ coupled to an external multiharmonic buncher was developed earlier and reported in Ref. [9]. In particular, it was shown that the new multisegment split-coaxial structure exhibits two distinguishing features: (1) a significant reduction of the transverse dimensions with respect to the four-vane structures and (2) a large frequency separation of neighboring nonoperational modes from the operational one. As was discussed in Ref. [9], the operational mode is the lowest mode in this structure and the nonoperational upper mode frequency is higher by $\sim 20 \%$. The relatively small transverse dimensions of the resonator allow the use of high-precision machining and high-temperature brazing techniques for fabrication. The frequency separation results in a stable accelerating field distribution that is not affected by manufacturing errors and frequency tuners.

The physics design of the RFQ must determine the exact cavity geometry, including vane tip modulations. Usually, the electromagnetic (EM) and beam dynamics of an RFQ are designed independently. For the ATLAS RFQ we developed a new, fully three-dimensional (3D) approach to model the full five-segment, 4-m-long ATLAS RFQ in Microwave Studio (MW-STUDIO) [10]. The main reason we decided to use a full 3D approach was to implement a trapezoidal vane tip modulation [11] in the acceleration section in order to increase the overall shunt impedance of the RFQ. The actual vane modulations were included so that we could study their effects on both frequency and field distribution [12]. We were also able to produce accurate 3D field maps to use in beam dynamics simulations. Integrating the electromagnetic and beam dynamics simulations in the same software provides a more consistent design evaluation. For the given energy gain, from $30 \mathrm{keV} / \mathrm{u}$ to approximately $300 \mathrm{keV} / \mathrm{u}$, the main design goals were as follows: (i) maximize the shunt impedance by EM optimization; (ii) determine the optimal RFQ length to minimize rf power loss and fabrication cost; (iii) form an axially symmetric beam upon exiting the RFQ; (iv) provide a total normalized transverse acceptance of $\sim 2 \pi \mathrm{mm} \mathrm{mrad}$; and (v) preserve the low longitudinal emittance of the multiharmonic buncher (MHB) and provide an acceleration efficiency higher than $80 \%$.

The geometry of the vane tip modulation was determined after several iterations between a simplified design using the RFQ design code DESRFQ [13] and the full 3D model of the RFQ in Electromagnetic Studio (EM-STUDIO) [14]. Because very few CW RFQs in the world are performing as designed, we adopted a very careful design and fabrication procedure. An interactive and iterative procedure between the different aspects of the design was implemented, namely, the electromagnetic design, beam dynamics simulations, engineering, and manufacturing.

Figure 1 shows the 3D model of the RFQ in MW-STUDIO. This model was created by using the engineering model and applying vane tip modulations. The basic parameters of the RFQ are listed in Table I.

\section{B. Trapezoidal vane tip modulation}

As presented in our previous work [10], the RFQ includes sinusoidal vane tip modulation in the bunching section and trapezoidal modulation in the accelerating section. This approach has been proposed and successfully implemented in a pulsed proton RFQ by the IHEP-Protvino group [11]. Adopting trapezoidal modulation in the accelerating section increased our RFQ output energy from 256 to $296.5 \mathrm{keV} / \mathrm{u}$ with minimal change in the rest of the beam dynamics. The trapezoidal modulation was not used in the bunching section for two reasons: (1) the original design from DESRFQ used sinusoidal modulation for the entire length and redesigning the whole RFQ with trapezoidal modulation was not practical; (2) the trapezoidal

TABLE I. Basic parameters of the RFQ.

\begin{tabular}{ll}
\hline \hline Parameter & Value \\
\hline Input energy & $30 \mathrm{keV} / \mathrm{u}$ \\
Output energy & $296.5 \mathrm{keV} / \mathrm{u}$ \\
Frequency & $60.625 \mathrm{MHz}$ \\
Vane voltage & $70 \mathrm{kV}$ \\
rf power calculated & $52 \mathrm{~kW}$ \\
by MW-STUDIO & \\
Average aperture radius & $7.2 \mathrm{~mm}$ \\
Length & $3.81 \mathrm{~m}$ \\
Transverse normalized & $2 \pi \mathrm{mm} \mathrm{mrad}$ \\
acceptance & \\
Longitudinal rms & $20 \pi \mathrm{deg} \cdot \mathrm{keV} / \mathrm{u}$ \\
emittance & $($ at $60.625 \mathrm{MHz})$ \\
& $0.9 \pi \mathrm{nsec} \cdot \mathrm{keV} / \mathrm{u}$ \\
Bunching & External 4-harmonic \\
\hline \hline
\end{tabular}




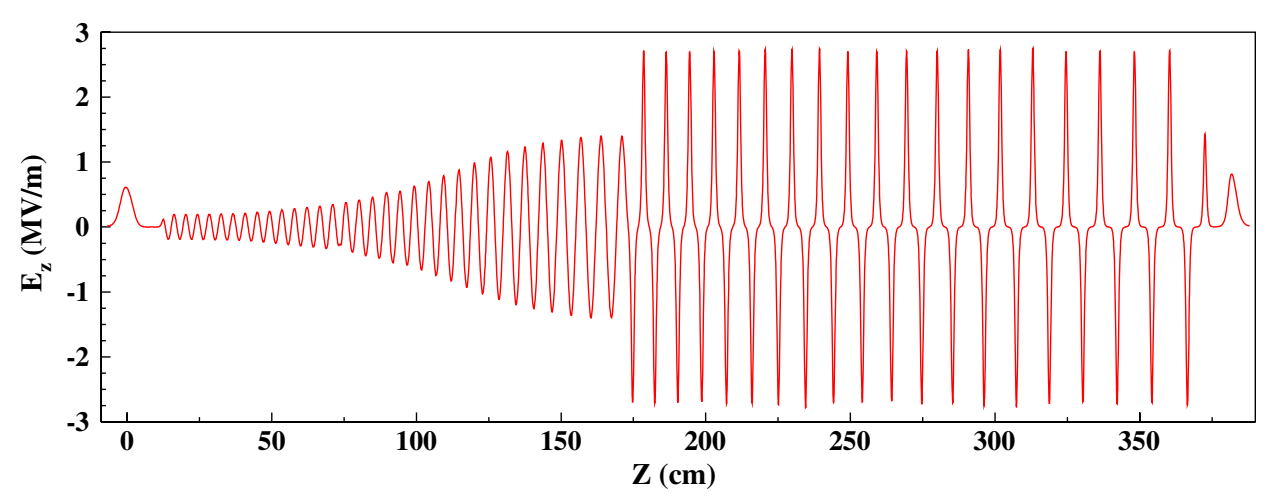

FIG. 2. On-axis longitudinal field along the RFQ.
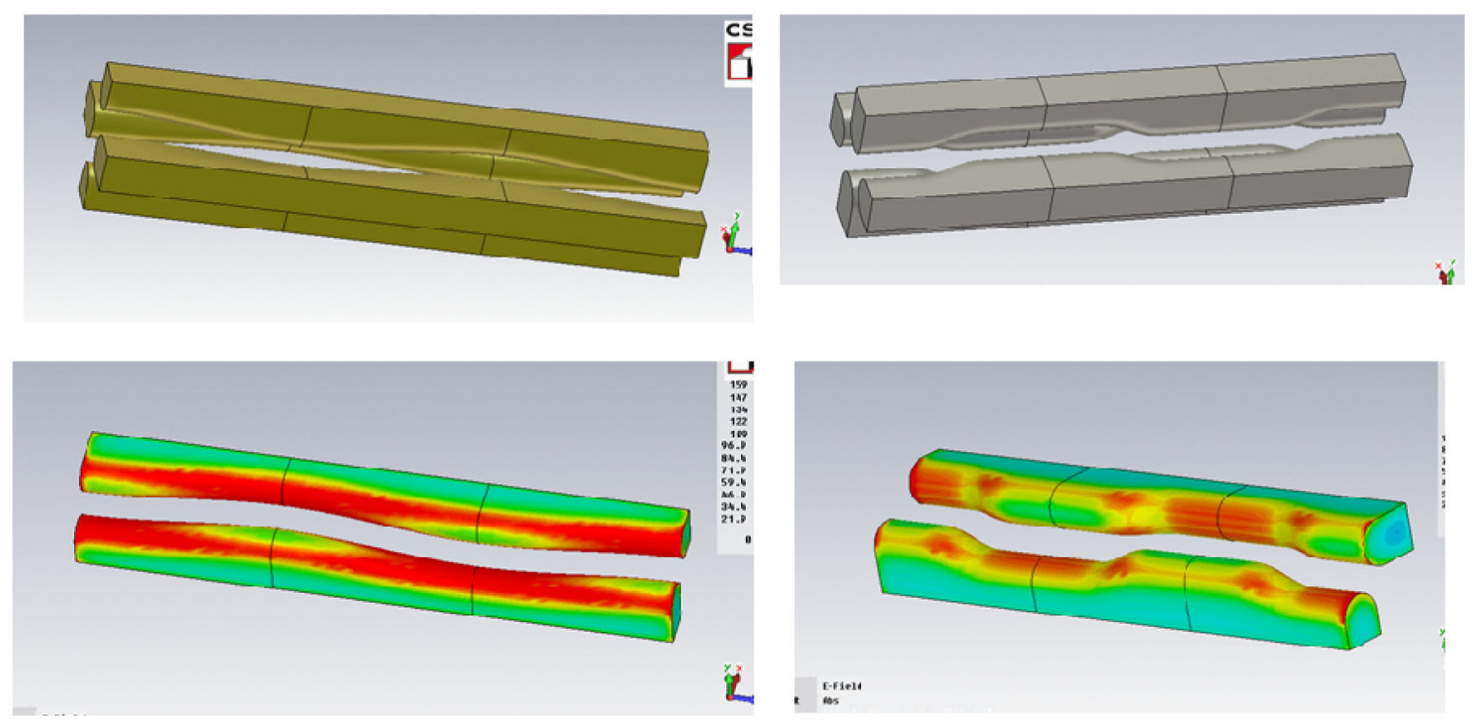

FIG. 3. Sinusoidal (left) and trapezoidal (right) vane tip modulations in EM-STUDIO models. The bottom pictures show surface electric field distribution.

modulation in the bunching section does not increase the shunt impedance. The EM simulations showed that the peak surface field in very small areas of the vane tip will increase by about $15 \%$ from the pure sinusoidal case but will remain below 1.8 Kilpatricks. It is worth recalling that the prototype $57.5-\mathrm{MHz}$ RFQ was tested up to 2.0 Kilpatricks without any voltage breakdowns [3]. Figure 2 shows the on-axis longitudinal field along the RFQ, clearly distinguishing the sinusoidal from the trapezoidal sections. Two comments should be made concerning the accelerating field distribution along the RFQ longitudinal axis. First, due to the split-coaxial structure there is a longitudinal electric field at both the entrance and the exit of the RFQ. As follows from the beam dynamics simulations, these fields do not affect beam quality. Second, the accelerating field appears immediately after the radial matcher because the RFQ starts with a $-50^{\circ}$ synchronous phase.

The full 3D field distribution was exported from MW-STUDIO [14] into TRACK [15] as a single cavity. TRACK simulations show excellent agreement between

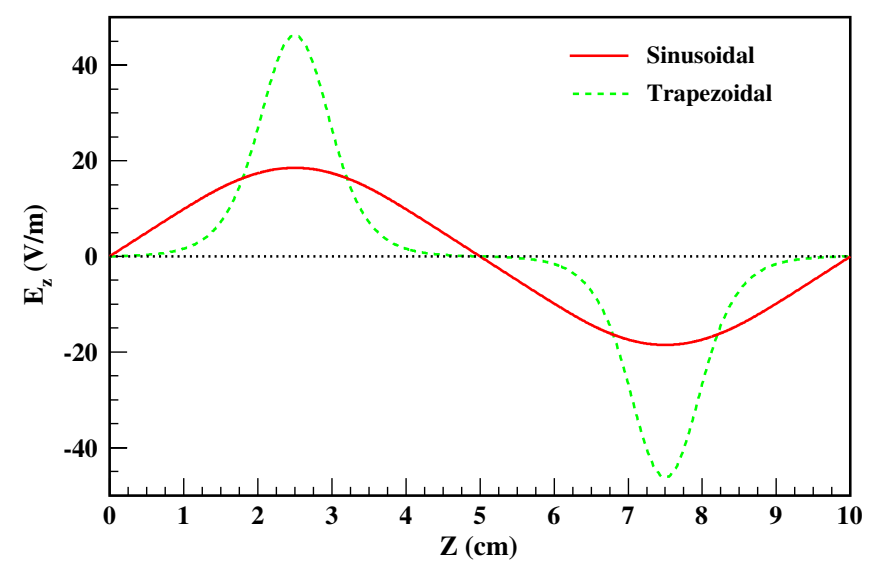

FIG. 4. Accelerating field distribution in the RFQ with sinusoidal (solid red line) and trapezoidal (dashed green line) modulations. 

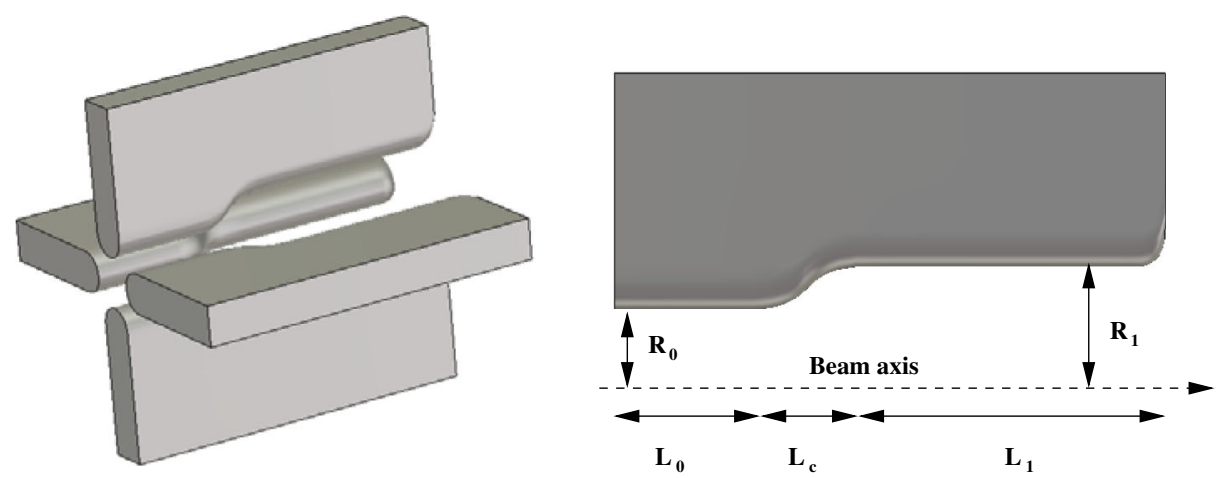

FIG. 5. Geometry of the new type of output radial matcher: A 3D view (left) and a 2D profile showing the parameters of the geometry (right).

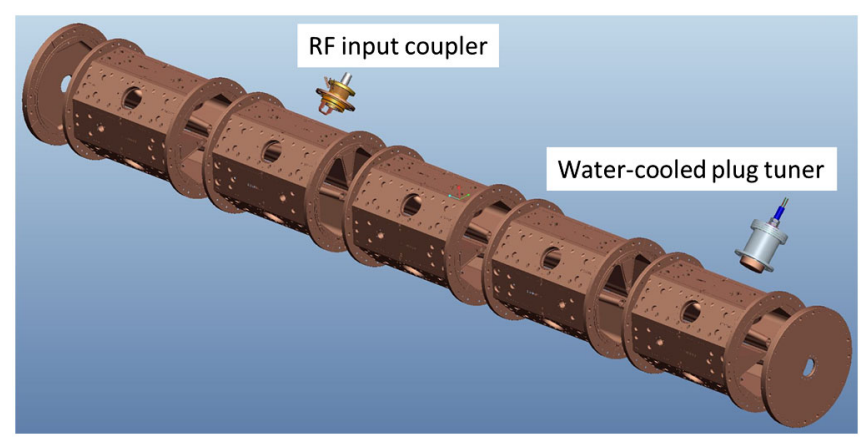

FIG. 6. 3D CAD model of the RFQ.

beam dynamics simulations in the RFQ with 3D field distribution from MW-STUDIO and the cell-by-cell 3D fields from EM-STUDIO [12].

The effective shunt impedance of the ATLAS RFQ is increased by $34 \%$ after introducing trapezoidal vane tip modulation instead of the traditional sinusoidal modulation in the accelerating section. Figure 3 shows the traditional sinusoidal and the new trapezoidal vane tip modulation. Typical accelerating field distributions for both types of vane modulations are shown in Fig. 4. Notice that the trapezoidal modulation modifies the field distribution significantly and increases the transit-time factor.

\section{Output radial matcher}

An output matcher is needed to produce an axially symmetric beam exiting the RFQ to match the beam to a subsequent solenoid-based focusing lattice. Instead of the more standard output matcher geometry obtained by mirroring the input radial matcher, we developed a new type of output matcher [10]. Figure 5 shows the geometry for the new matcher. It consists of an initial straight section of length $L_{0}$ with an average radius $R_{0}$, followed by a curved section of length $L_{c}$ and a final straight section of length $L_{1}$ at a radius $R_{1}$. Only the average radius $R_{0}$ and the total matcher length $L_{0}+L_{c}+L_{1}$ are defined because of the limited RFQ length. The other parameters, $L_{0}, L_{1}$, and $R_{1}$, are determined by optimization to produce an axially

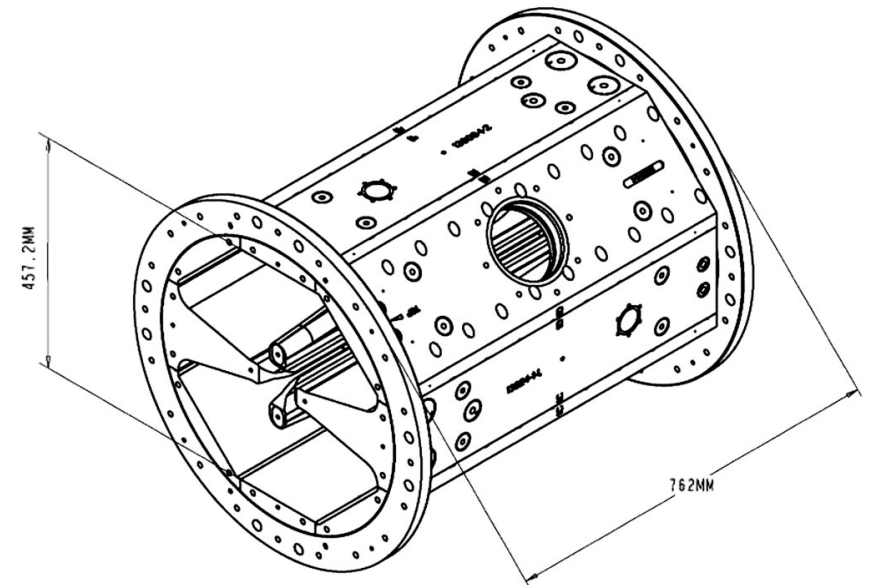

FIG. 7. CAD model of the first segment.

symmetric beam at the RFQ exit. A total matcher length of $0.75 \beta \lambda$ was enough to produce a symmetric beam.

\section{Mechanical design and engineering analysis}

The RFQ resonator consists of five 30-inch-long segments, as shown in Fig. 6. The RFQ coupler, also shown

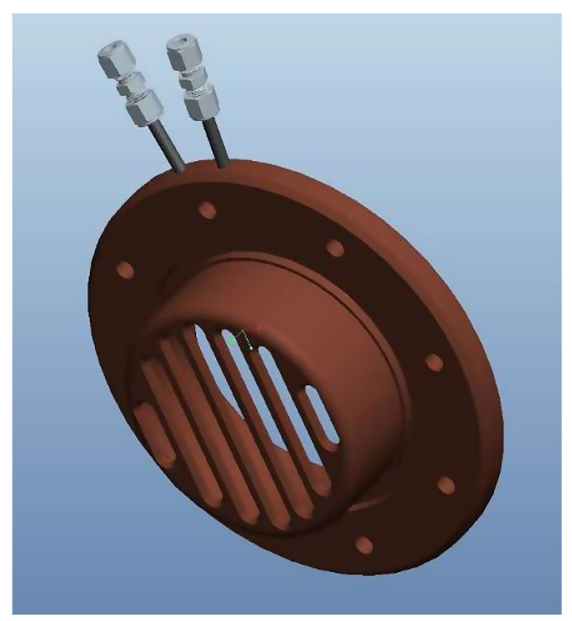

FIG. 8. 3D model of the water-cooled "vacuum grill." 


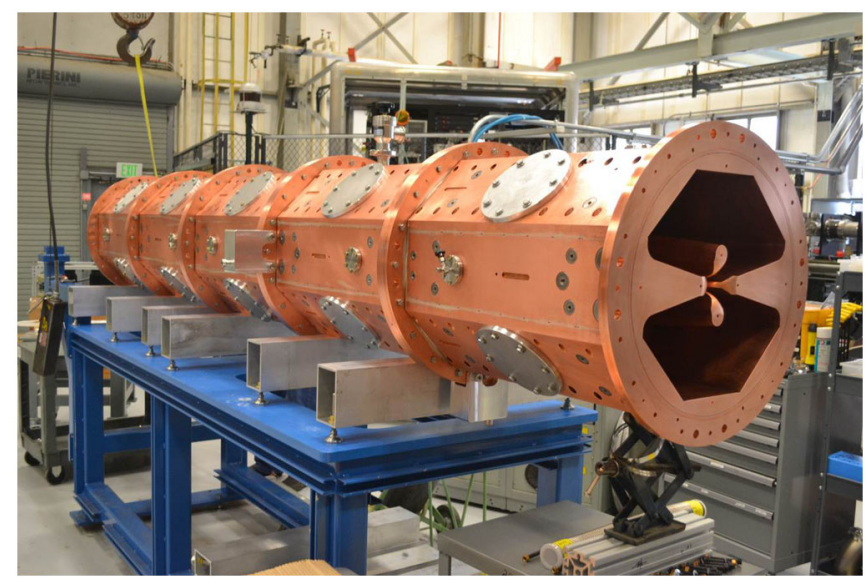

FIG. 9. Completed assembly of the five-segment RFQ.

in Fig. 6, has the same design as the prototype onesegment 57.5-MHz RFQ [3]. The computer-aided design (CAD) model of the first segment is shown in Fig. 7. The five segments are nearly identical except for the vane tip modulation. One of the mating surfaces between the segments is equipped with grooves for canted coil rf springs [16] and a Viton O-ring. Each segment has four 5-inch ports for the installation of frequency tuners, rf couplers, and vacuum pumps. The frequency tuner is a water-cooled copper plug (Fig. 6) with a 3-inch stroke. There are four $1 \frac{5}{8}$-inch ports in each segment for pickup loops, vacuum gauges, and a venting flange. The rf currents between all mechanical joints are shielded by canted coil rf springs. The specified machined accuracy of all parts was $25 \mu \mathrm{m}$ and was confirmed with measurements. Five $650 \mathrm{l} / \mathrm{s}$ turbopumps are used for pumping through the water-cooled "vacuum grill" (see Fig. 8) installed in designated 5-inch ports.

Modeling and multiphysics analyses of the RFQ resonator were performed using the ANSYS software package [17]. The heat loads were determined from the magnetic fields at full power. The heat loads were then transferred to a thermal model to determine temperatures in the presence of water cooling. The temperature distribution was then input into a structural model to determine displacements and stresses, and then the displacements were input back into the rf model to get the frequency shift. The final design

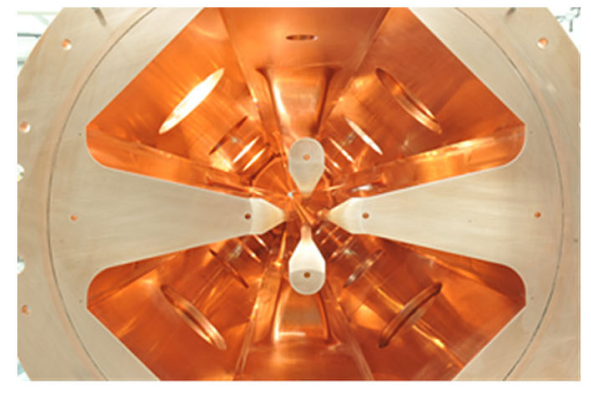

results in vane tip displacements of $58 \mu \mathrm{m}$ near the center of the segments at full-power operation. These small deflections of the vane tips were obtained by optimizing the locations and paths of the cooling channels [18].

\section{E. Resonance control cooling system}

The RFQ resonant frequency is regulated by dynamically adjusting the water temperature in the body of the RFQ while the temperature of the vanes is kept constant. Similarly, the control system can use the water temperature of the vanes in the frequency feedback system while keeping the body temperature constant. The exact method for frequency control will be established experimentally during on-line operation, which requires setting different RFQ power levels for the acceleration of different ion species. A mixer adjusts the water temperature by combining chilled water from the external cooling system and hot water returning from the resonator. The resonance control cooling system has been built and successfully tested at $\mathrm{rf}$ power levels up to $20 \mathrm{~kW}$. A detailed description of this system is given in Ref. [19].

\section{FABRICATION}

The RFQ is designed as a $100 \%$ OFE copper structure including flanges and end caps. The fabrication process is the same for the prototype one-segment 57.5-MHz RFQ [3] and includes the following main stages: (i) delivery of raw copper (copper samples are checked for low oxygen content); (ii) preliminary machining of vanes, quadrants, and end caps; drilling of water-cooling channels; and hightemperature furnace brazing of water channel plugs using a 35-65 $\mathrm{Au}-\mathrm{Cu}$ alloy in a hydrogen atmosphere; (iii) hydrostatic pressure testing of the cooling channels; (iv) final machining of all parts, including vane tip modulation and cleaning in a heated Citranox bath; (v) fabrication of the fixture required for segment assembly, lifting, and transportation; fabrication of the cavity support fixture to be used in the furnace; (vi) assembly of each segment; prebraze machining to install end flanges; frequency check of individual segments; disassembly of segments and cleaning of all parts in a heated Citranox bath; assembly and preparation to load into the furnace in vertical orientation; (vii) final brazing using CuSil alloy in

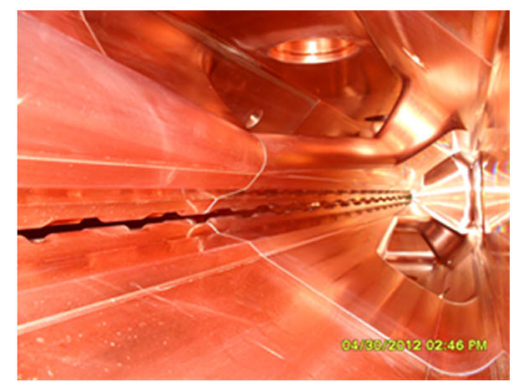

FIG. 10. Internal view of the RFQ from the low-energy end (left) and from the third segment to the high-energy end (right). 
TABLE II. Comparison of the simulated and measured parameters of the RFQ resonator. The design simulations were originally done in 2010 using the hexahedral mesh option in MW-STUDIO. In 2011, the tetrahedral mesh option became available and repeated simulations led to new results that were different from the original simulations but much closer to the measured data.

\begin{tabular}{lcccc}
\hline \hline Parameter & \multicolumn{2}{c}{ Simulation (no tuners) } & \multicolumn{2}{c}{ Measurements } \\
& CST-2010 hexahedral & CST-2011 tetrahedral & No tuners & Tuners inserted \\
\hline Frequency, MHz & 60.267 & 60.150 & 60.064 & 60.625 \\
Quality factor & 10057 & 9873 & 9300 & 8600 \\
Stored energy $\left(V_{0}=70 \mathrm{kV}\right), \mathrm{J}$ & 1.270 & 1.330 & 1.330 \\
rf power to obtain $V_{0}=32.0 \mathrm{kV}, \mathrm{kW}$ & 9.99 & 10.65 & 12.5 \\
rf power to obtain $V_{0}=70 \mathrm{kV}, \mathrm{kW}$ & 47.79 & 50.94 & 59.7 \\
\hline \hline
\end{tabular}

a hydrogen retort atmosphere furnace; (viii) postbrazing final machining; rf measurements and final cleaning of segments in a heated Citranox bath; vacuum leak check of segments; (ix) assembly of the segments and end flanges; installation of external water-cooling pipes, water-cooled tuners, pickup loops, driving loops, rf transmission line, and vacuum system components.

Coordinate measuring machine (CMM) measurements of all resonator components have been performed at various stages of the manufacturing process. Final CMM measurements of the vane tip-to-tip distances at the segment ends showed that vane positions are within $\pm 25 \mu \mathrm{m}$ of the design values. The vane tip modulations were machined per design dimensions without any noticeable errors. The longitudinal dimensions of the 30-inch segments were within $\pm 12.5 \mu \mathrm{m}$. The measured frequencies of individual segments prior to final brazing were very close to the MWSTUDIO simulated frequencies with deviations of -25 to $+91 \mathrm{kHz}$.

\section{ASSEMBLY AND RF TUNING}

All five segments of the RFQ were assembled horizontally, directly on the support table as shown in Fig. 9. Four pins on the flanges guided the segments together during assembly. The assembled RFQ resonator was lifted and installed on the kinematic mount. Figure 10 shows internal views of the RFQ, looking from the entrance and from the third segment down to the RFQ end. The trapezoidal vane tip modulation is clearly evident in the last three segments. The next step was the installation of 12 tuners, 2 rf couplers, 8 pickup loops, and 5 turbopumps. All pickup loops were identical. Five calibrated pickup loops provide information about the uniformity of the magnetic field level in each segment. In the final on-line installation, two amplifiers will provide the rf power into the RFQ through two identical rf couplers. The two rf couplers will be tuned to provide a loaded $Q_{L}=\frac{1}{2} \cdot Q_{0}$, where $Q_{0}$ is the intrinsic quality factor of the RFQ cavity. During off-line beam commissioning, the RFQ is operated at lower power up to $15 \mathrm{~kW}$, transmitted through a single rf coupler tuned for critical coupling.

The RFQ resonator was designed to have a $60.25-\mathrm{MHz}$ resonant frequency when all tuners and vacuum grills are inserted to the depth of the resonator wall thickness, creating even internal surfaces. The approximately $0.4-\mathrm{MHz}$ difference between the operational and design frequencies is a safety margin to ensure that the RFQ can be tuned to the operational frequency. Fully inserted, the 12 tuners can increase the RFQ frequency by approximately $0.8 \mathrm{MHz}$, enough to cover the safety margin and eventual simulation and fabrication errors. The measured frequency was slightly lower than the simulated one, as shown in Table II, and the final frequency tuning was performed by inserting six tuners by 1 inch and another six tuners by 1.75 inches into the resonator rf volume. As seen from Table II, the intrinsic $Q$ factor was $5.8 \%$ lower than the MWS-calculated value, and the insertion of the tuners further reduced the quality factor. Therefore, the required rf power is $59.7 \mathrm{~kW}$, which is $13 \%$ higher than the MWScalculated value for the resonator without tuners.

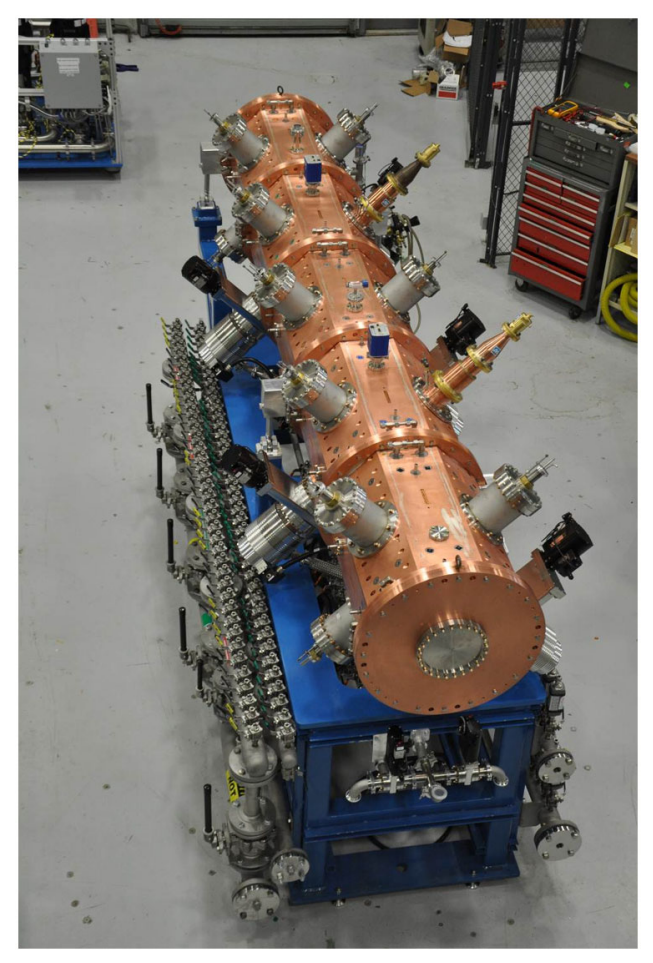

FIG. 11. RFQ assembly after installation of tuners, $r f$ couplers, pickup loops, vacuum pumps, and vacuum gauges. 

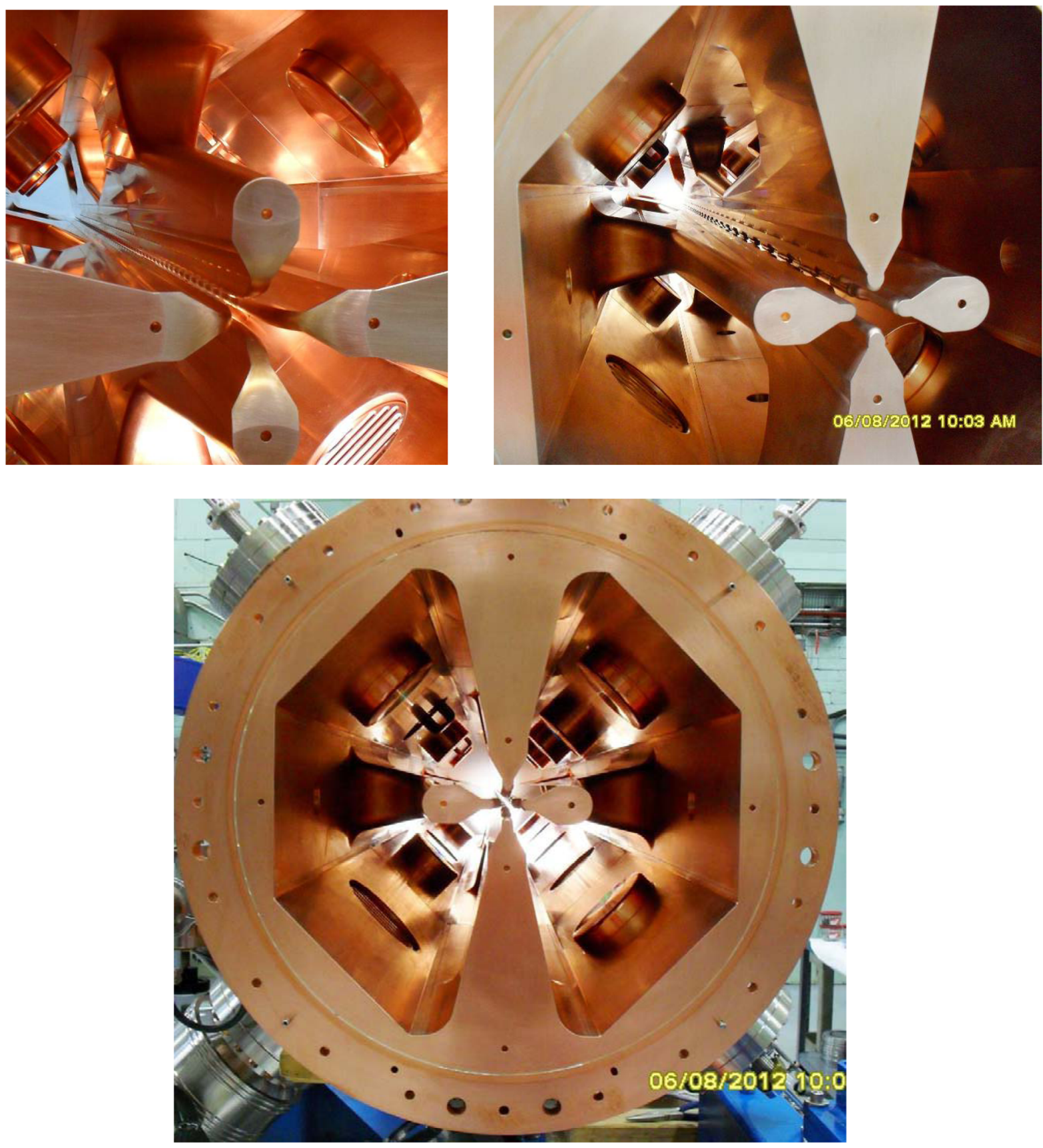

FIG. 12. Internal views of the RFQ after completed assembly. View from the low-energy end (top left) and high-energy end (top right and bottom).

The power attenuation through the driving loop to the designated pickup loop was measured by an HP Network Analyzer, and was equal to $50.047 \mathrm{~dB}$. The intervane voltage can be set by measuring the rf power from the calibrated pickup loop. For example, a pickup loop power of $119 \mathrm{~mW}$ corresponds to $32 \mathrm{kV}$, the design voltage for acceleration of ${ }^{16} \mathrm{O}^{5+}$ ions, and $570 \mathrm{~mW}$ corresponds to $70 \mathrm{kV}$, the design voltage for acceleration of ions with $1 / 7$ charge-to-mass ratio. Finally, Fig. 11 shows the RFQ assembly after installation of the tuners, vacuum pumps, vacuum gauges, rf couplers, and pickup loops. Internal views of the RFQ after complete assembly clearly showing plug tuners, rf couplers, and vacuum grills are presented in Fig. 12.

\section{EXPERIMENTAL SETUP}

The RFQ was connected to the existing permanent magnet electron cyclotron resonance ion source (PM ECRIS) through the low-energy beam transport (LEBT) line. Both the PM ECRIS and the LEBT were used in our 


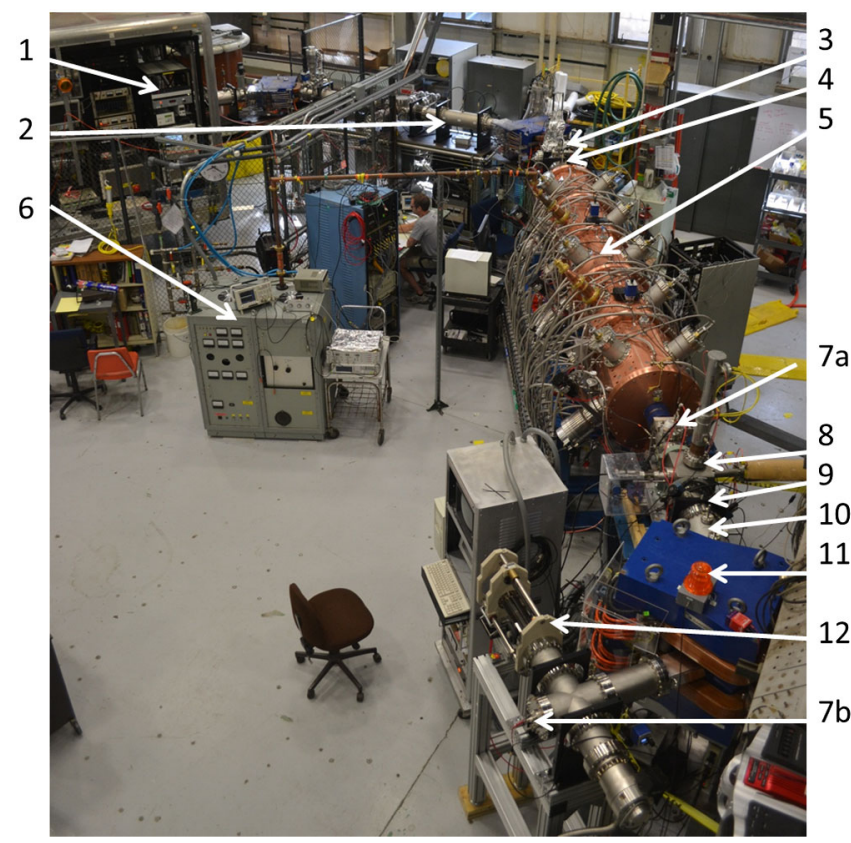

FIG. 13. General view of the RFQ test area. 1-All permanent magnet ECRIS installed on HV platform; 2-LEBT; 3 - pepperpot emittance probe; 4-matching quadrupole triplet; 5-RFQ; 6 -rf amplifier; 7a, 7b-water-cooled Faraday cup; 8-bunch shape monitor; 9-rotating wire scanner; 10 -electrostatic doublet; $11-70^{\circ}$ bending magnet; 12 -water-cooled movable horizontal jaw slits.

previous research work and were described in Ref. [20]. The experimental setup used for off-line beam commissioning of the RFQ is shown in Fig. 13. It consists of a PM ECRIS, a $100-\mathrm{kV}$ platform, an achromatic LEBT system based on two $60^{\circ}$ bending magnets, several Faraday cups, a pepper-pot emittance probe, a matching electrostatic triplet, the RFQ, a bunch shape monitor, a rotating wire scanner, an electrostatic doublet, a $70^{\circ}$ bending magnet, and movable slits. All Faraday cups and slits after the RFQ are water cooled.

The RFQ is designed for acceleration of prebunched beams formed by an external four-harmonic buncher (the
MHB) to provide a very small longitudinal emittance with 83\% acceleration efficiency. The MHB is not available in the off-line installation of the RFQ, so testing was performed with DC ion beams.

\section{A. $r$ conditioning}

A narrow-band $15-\mathrm{kW}$ rf amplifier tuned to a central frequency of $60.625 \mathrm{MHz}$ and two $20-\mathrm{kW}$ water chillers were available for the off-line RFQ testing. A low level radio frequency control module was developed to operate the RFQ in a self-excited mode. The rf conditioning was started at an RFQ resonator vacuum level of $4 \times$ $10^{-7}$ Torr. When we first turned on the rf power to approximately $1 \mathrm{~kW}$, we observed a reflected power at the level of 0.5 to $1 \mathrm{~kW}$, indicating some discharge activities. After about 30 minutes of conditioning, the full available power up to $15 \mathrm{~kW}$ was transmitted into the resonator. A meter directly measured the calibrated pickup loop power to calibrate the intervane voltage. The resonator vacuum is continuously improving and after 2 months of pumping, the pressure is better than $7 \times 10^{-8}$ Torr.

\section{B. Beam measurements}

The total voltage of the ECR and high voltage (HV) platform was set to $96 \mathrm{kV}$ to provide the $30-\mathrm{keV} / \mathrm{u}$ design injection energy for the ${ }^{16} \mathrm{O}^{5+}$ test beam. The ECR and $60^{\circ}$ bending magnets were tuned to transport approximately $40 \mu \mathrm{A}$ of ${ }^{16} \mathrm{O}^{5+}$ ions to the RFQ entrance. Figure 14 shows a typical emittance measurement taken by the pepper-pot emittance probe [21] upstream of the electrostatic triplet. The measured Twiss parameters were used to match the beam to the RFQ acceptance using the electrostatic triplet, which is directly attached to the entrance flange of the RFQ. The calculated beam rms envelopes between the emittance probe (number 3 in Fig. 13) and Faraday cup (number $7 b$ in Fig. 13) are shown in Fig. 15.

Several beam diagnostic devices were installed downstream of the RFQ to measure the main beam parameters: (1) a water-cooled retractable Faraday cup to measure beam transmission through the RFQ (number $7 \mathrm{a}$ in
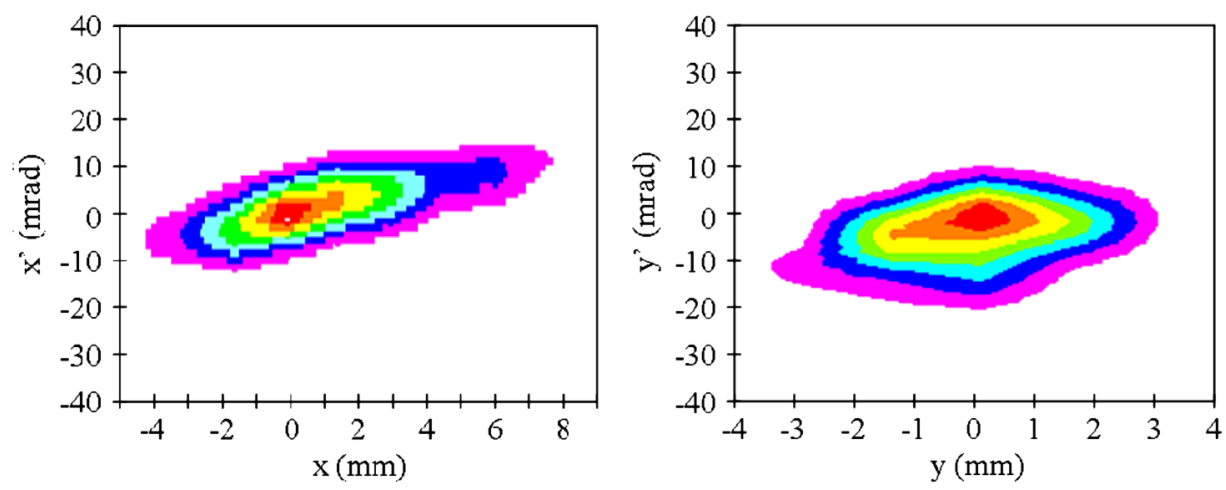

FIG. 14. Transverse beam phase space upstream of the RFQ. 


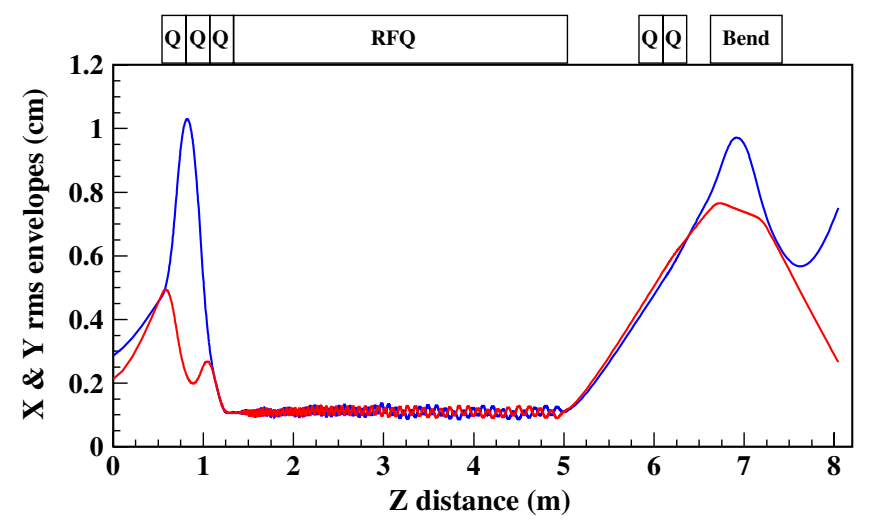

FIG. 15. Calculated beam rms envelopes in the section from the emittance probe to the high-dispersion image plane ( $X$ : blue line; $Y$ : red line) for an rms-normalized emittance of $0.083 \pi \mathrm{mm}$ mrad.

Fig. 13); (2) a bunch shape monitor; (3) a rotating wire scanner (number 9 in Fig. 13); and (4) movable jaw slits followed by a stationary Faraday cup. The jaw slits with a 0.2-mm gap are installed in the high-dispersion plane after the magnet. To achieve the best resolution for the energy spread measurements, the electrostatic lens (number 10 in Fig. 13) was adjusted to obtain a Twiss parameter $\alpha=0$ at the high-dispersion image plane for the trial monochromatic beam. Figure 16 shows the $(x, \Delta \mathrm{W})$ phase space of the simulated beam in the image plane. The combination of the electrostatic quadrupole and bending magnet with edge focusing creates a very small beta function, $\beta_{x}=$ $4.5 \mathrm{~mm} / \mathrm{mrad}$ in the high-dispersion image plane. The energy resolution is $0.5 \mathrm{keV} / \mathrm{u}$ for the $0.1 \pi \mathrm{mm} \times \mathrm{mrad}$ beam rms-normalized emittance.

The first experiment was to measure the beam transmission through the RFQ as a function of the rf power. Beam current was measured by retractable Faraday cups

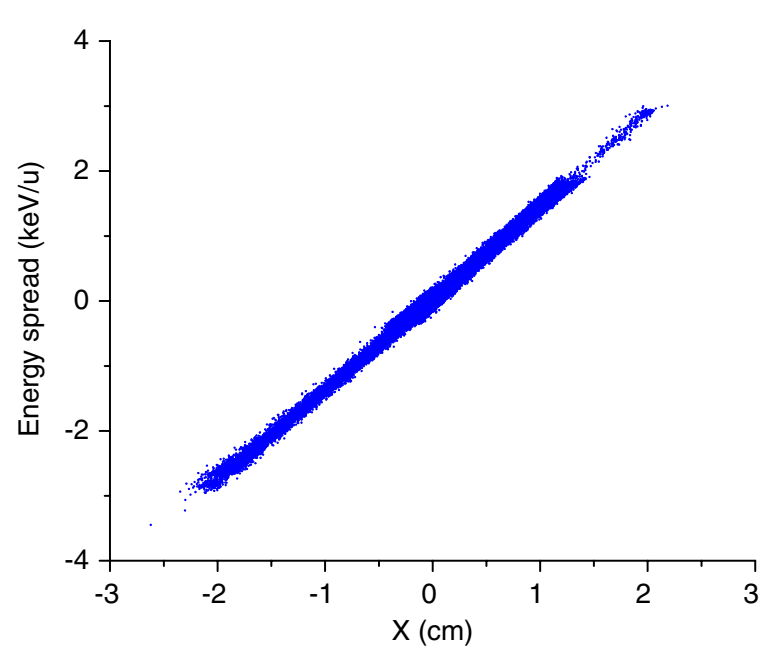

FIG. 16. Simulated beam in the $(x, \Delta \mathrm{W})$ phase space at the image plane. upstream and downstream of the RFQ. The corresponding data compared to TRACK simulation results are shown in Fig. 17. A four-dimensional (4D) Gaussian DC beam with an rms-normalized emittance of $0.083 \pi \mathrm{mm} \mathrm{mrad}$ at the RFQ entrance corresponding to the pepper-pot measurements shown in Fig. 14 was used in the simulations.

The RFQ is designed to form an axially symmetric beam. To confirm this property of the RFQ, we installed a 40-mm aperture rotating wire scanner $98 \mathrm{~cm}$ downstream of the RFQ (see Fig. 13). Using the beam optics $\beta$ function for the matched beam from TRACK simulations, the beam rms emittance can be calculated from the profile measurements. For these measurements, the RFQ was set to the design $32-\mathrm{kV}$ intervane voltage required for the acceleration of the ${ }^{16} \mathrm{O}^{5+}$ beam. Figure 18 shows the superimposition of the measured and simulated beam profiles. The simulated data corresponds to a transverse rms emittance of $0.105 \pi \mathrm{mm}$ mrad at the RFQ entrance. In the measurements we observe about a 2-mm beam center shift in the vertical plane. To compare the measured data to the simulation, we ignored the beam center shift and renormalized the data to the same integral, $\int_{-\infty}^{\infty}[f(u)-f(\hat{u})] d u$, where $f(u)$ is the beam intensity distribution, $\hat{u}$ is the measured beam center, and $u=x, y$.

The beam energy spread was determined by measuring the beam current with the Faraday cup located behind the slits while scanning the magnetic field level in the $70^{\circ}$ bending magnet. The results of these measurements are shown in Fig. 19. To compare the simulated curve to the measured data, we renormalized to the same area under the curves and applied a global shift of approximately $2 \%$ to match the location of the peaks. The average beam energy corresponds to the measured magnetic field of $6.825 \mathrm{kGs}$. The magnetic field was measured between the poles in the central region using a Hall probe with $2 \%$ accuracy. Detailed ion trajectory calculations in a $3 \mathrm{D}$ map of the

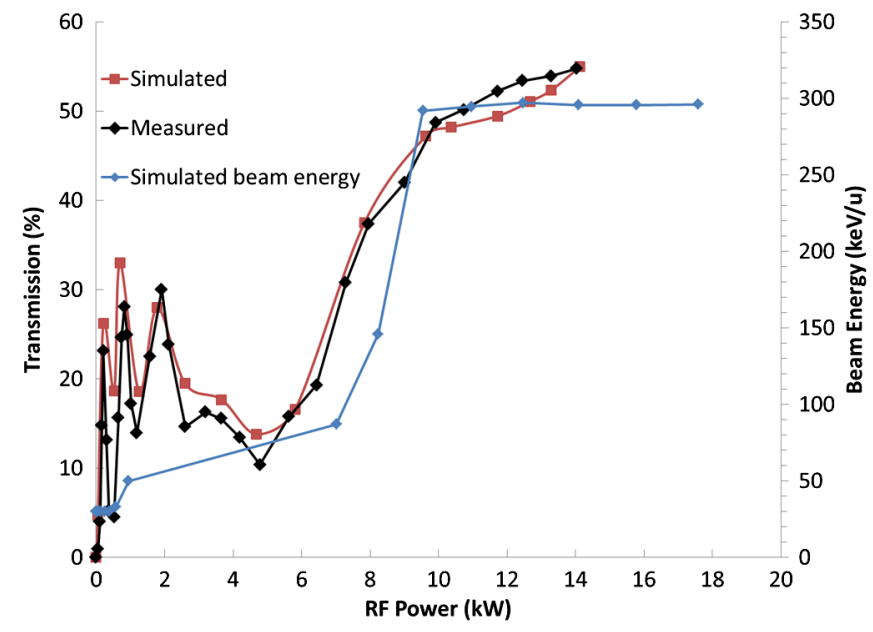

FIG. 17. Measured and simulated beam transmission as a function of $\mathrm{rf}$ power along with the simulated beam average energy. 

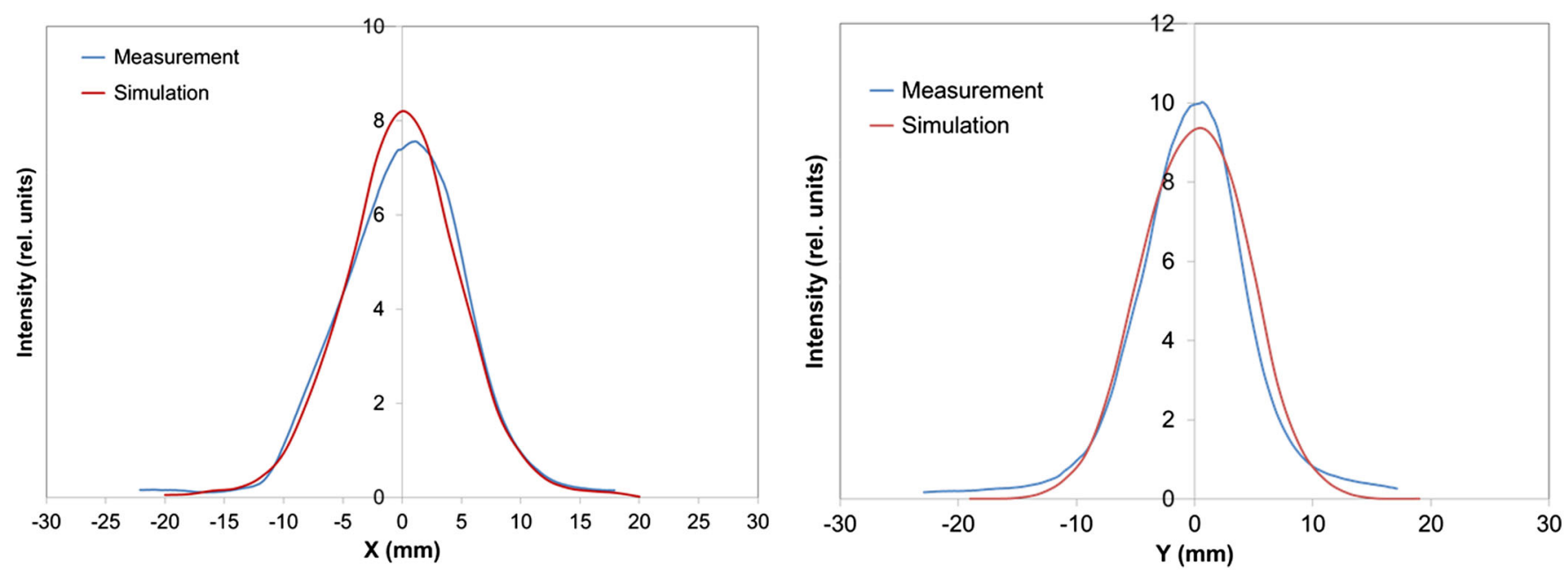

FIG. 18. Measured and simulated beam profiles at $98 \mathrm{~cm}$ downstream of the RFQ.

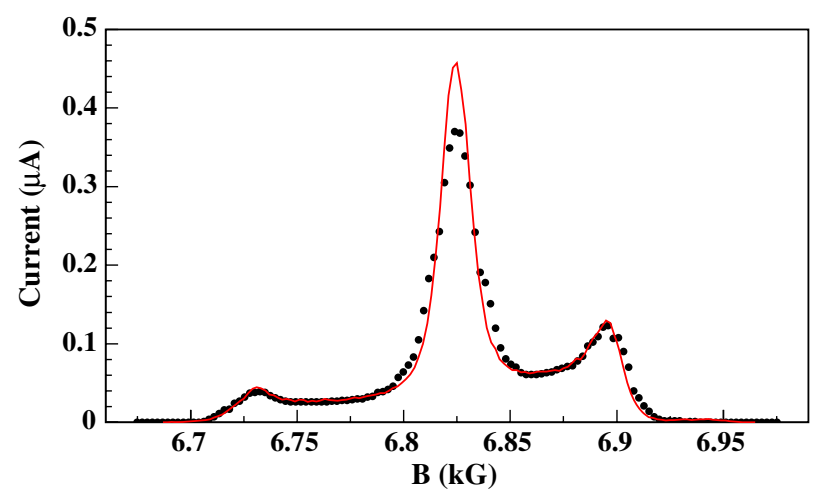

FIG. 19. Measured (dots) and simulated (red curve) beam intensity as a function of the magnetic field.

magnetic field confirmed that the calculated and measured beam energies are equal to each other within $2 \%$ error.

\section{Discussion of the results}

The comparison plots in Figs. 17-19 show the excellent agreement of the simulated and measured results, namely: (i) the measured beam average energy is equal to the simulated one: $296.5 \mathrm{keV} / \mathrm{u}$ with an accuracy of $\sim 6 \mathrm{keV} / \mathrm{u}$; (ii) the RFQ forms an axially symmetric beam as designed; (iii) no rms emittance growth is observed for the accelerated beam in the RFQ; and (iv) the energy spread in the beam is nearly identical to the simulated one.

\section{SUMMARY}

We have designed, constructed, and beam tested a CW RFQ. Several innovative design concepts and fabrication procedures were implemented in this RFQ. For the design of the RFQ resonator and vane tip modulations we devel- oped a full 3D approach that includes MW-STUDIO and TRACK simulations of the entire structure. The multisegment split-coaxial structure creates strong coupling between the quadrants and individual RFQ segments, which reduces the effect of local frequency deviations on electromagnetic field distortions. Therefore, no bead-pull measurements were required for tuning of the accelerating field. A novel trapezoidal vane tip modulation is used in the acceleration section of the RFQ, which resulted in increased shunt impedance. We also developed a short output radial matcher to form an axially symmetric beam exiting the RFQ.

An advanced fabrication technology was applied for the construction of the RFQ that includes precise machining and two-step high-temperature brazing. Thanks to the high accuracy of the overall fabrication, the assembly of the five-segment RFQ was straightforward and resulted in excellent alignment. The resonance frequency control system based on water temperature regulation showed excellent performance. After 2 months of pumping, the RFQ vacuum achieved $6 \times 10^{-8}$ Torr despite using Viton $\mathrm{O}-$ rings in all vacuum joints. The great success of this advanced design and fabrication technology is reflected by the measured beam parameters after the RFQ, which are nearly identical to the simulated data.

Full-power testing of the RFQ is planned for November 2012, followed by integration into ATLAS.

\section{ACKNOWLEDGMENTS}

Many engineers, technicians, and machinists from several vendors contributed to the overall success of the RFQ fabrication and beam testing. In particular, we would like to thank the following key contributors: S. Kutsaev and K. Miller (Argonne, Physics); W. Jansma and B. Rusthoven (Argonne, Advanced Photon Source); G. Cherry (Argonne, Nuclear Engineering); F. Hunt and 
R. Reierson (Argonne, Facilities Management and Services); E. Biniak, N. Spader, R. Myrda, and B. Lattina (Walco Tool); C. Tomatz (Carlson Tool); I. Heber and B. Houghtelling (CalBraze); and D. Raska (Weldaloy Products). This work was supported by the U.S. Department of Energy, Office of Nuclear Physics, under Contract No. DE-AC02-06CH11357.

[1] P. N. Ostroumov, R. V.F. Janssens, M.P. Kelly, S. A. Kondrashev, B. Mustapha, R. C. Pardo, and G. Savard, in Proceedings of LINAC-10, Tsukuba, Japan, 2010 [http://www.jacow.org], p. 157.

[2] P. N. Ostroumov, A. Barcikowski, Z. A. Conway, S. M. Gerbick, M. Kedzie, M.P. Kelly, S. W. T. MacDonald, B. Mustapha, R.C. Pardo, and S.I. Sharamentov, in Proceedings of the 2011 Particle Accelerator Conference, NY, USA (IEEE, New York, 2011), p. 2110.

[3] P. N. Ostroumov, A. Barcikowski, B. M. Rusthoven, S. I. Sharamentov, S. Sharma, W. F. Toter, J. Rathke, D. L. Schrage, and N. Vinogradov, in Proceedings of LINAC06, Knoxville, TN, 2006 [http://www.jacow.org], p. 767.

[4] L. M. Young, in Proceedings of the 19th Particle Accelerator Conference, Chicago, Illinois, 2001 (IEEE, Piscataway, NJ, 2001), p. 309.

[5] R. L. Poirier, R. Baartman, P. Bricault, K. Fong, S. Koscielniak, R. Laxdal, A. K. Mitra, L. Root, G.m. Stanford, and D. Pearce, in Proceedings of the 20th International Linac Conference, Monterey, CA, 2000 (SLAC, Menlo Park, CA, 2000), p. 1023.

[6] V.P. Derenchuk, V. Anferov, G. East, D. L. Friesel, R. W. Hamm, W. P. Jones, and J. Staples, in Proceedings of the 21st Particle Accelerator Conference, Knoxville, 2005 (IEEE, Piscataway, NJ, 2005), p. 3179.

[7] N. Sakamoto, in Proceedings of LINAC-12, Tel-Aviv, Israel, 2012 [http://www.jacow.org].
[8] L. Weissman, D. Berkovits, I. Eliyahu, I. Gertz, A. Grin, S. Halfon, G. Lempert, I. Mardor, A. Nagler, A. Perry, J. Rodnizki, K. Dunkel, M. Pekeler, C. Piel, and P. vom Stein, in Proceedings of LINAC-10, Tsukuba, Japan, 2010 [http://www.jacow.org], p. 157.

[9] P. N. Ostroumov, A. A. Kolomiets, D. A. Kashinsky, S. A. Minaev, V.I. Pershin, T.E. Tretyakova, and S.G. Yaramishev, Phys. Rev. ST Accel. Beams 5, 060101 (2002).

[10] B. Mustapha, A. Kolomiets, and P. N. Ostroumov, in Proceedings of LINAC-10, Tsukuba, Japan, 2010 [http:// www.jacow.org], p. 542.

[11] O. K. Belyaev, O. V. Ershov, I. G. Maltsev, V. B. Stepanov, S. A. Strekalovskikh, V.A. Teplyakov, and A. V. Zherebtsov, in Proceedings of the 20th International Linac Conference, Monterey, CA, 2000 (SLAC, Menlo Park, CA, 2000), p. 259.

[12] B. Mustapha, A. Kolomiets, and P. N. Ostroumov, in Proceedings of the 2011 Particle Accelerator Conference, NY, USA (IEEE, New York, 2011), p. 97.

[13] The RFQ design software DESRFQ, A. A. Kolomiets et al., ITEP/ANL Technical Note, 2005.

[14] CST EM-STUdIO and MW-STUDIO, CST, GmbH, Darmstadt, Germany [http://www.cst.de].

[15] The beam dynamics code TRACK [http://www.phy.anl.gov/ atlas/TRACK].

[16] Available at http://www.NPIsprings.com.

[17] Available at http://www.ansys.com.

[18] T. Schultheiss, J. Rathke, A. Barcikowski, P. N. Ostroumov, and D.L. Schrage, in Proceedings of the 2011 Particle Accelerator Conference, NY, USA (IEEE, New York, 2011), p. 1334.

[19] S. I. Sharamentov, in Proceedings of LINAC-12, Tel-Aviv, Israel, 2012 [http://www.jacow.org].

[20] P. N. Ostroumov, S. A. Kondrashev, B. Mustapha, and R. Scott, Phys. Rev. ST Accel. Beams 12, 010101 (2009).

[21] S. Kondrashev, A. Barcikowski, B. Mustapha, P. N. Ostroumov, and N. Vinogradov, Nucl. Instrum. Methods Phys. Res., Sect. A 606, 296 (2009). 